Research Article

\title{
Heat and Mass Transfer in Stagnation Point Flow of Maxwell Nanofluid Towards a Vertical Stretching Sheet with Effect of Induced Magnetic Field
}

\author{
Tadesse Walelign (iD, Eshetu Haile $(\mathbb{D}$, Tesfaye Kebede, and Gurju Awgichew \\ Department of Mathematics, Bahir Dar University, P.O. Box 79, Bahir Dar, Ethiopia \\ Correspondence should be addressed to Tadesse Walelign; tadelenyosy@gmail.com
}

Received 8 November 2020; Revised 26 February 2021; Accepted 6 March 2021; Published 17 March 2021

Academic Editor: Luigi Rodino

Copyright (c) 2021 Tadesse Walelign et al. This is an open access article distributed under the Creative Commons Attribution License, which permits unrestricted use, distribution, and reproduction in any medium, provided the original work is properly cited.

\begin{abstract}
This paper presents a mathematical model analysis of heat and mass transfer in a two-dimensional flow of electrically conducting, thermally radiative, and chemically reactive Maxwell nanofluid towards a vertical stretching and permeable sheet embedded in a porous medium. Boundary layer approximation and suitable transformations are used to reduce the governing differential equations convenient for computation. Eventually, the transformed nonlinear differential equations along with the corresponding boundary conditions are solved in the framework of optimal homotopy analysis method. The effects of induced magnetic field, buoyancy force, viscous dissipation, heat source, Joule heating, and convective boundary condition are analyzed in detail. The rates of heat, mass, and momentum transfer with respect to the relevant parameters are also examined in terms of the local Nusselt number, Sherwood number, and skin friction coefficients, respectively. Among the many results of the study, it is shown that the induced magnetic field, flow velocity, and temperature profiles are increasing functions of the Maxwell parameter. The results of the present study are also in a close agreement with previously published results under common assumptions.
\end{abstract}

\section{Introduction}

Based on the deformation of fluids in response to the applied shear stress, fluids can be classified into two categories, namely, Newtonian and non-Newtonian. Fluids such as honey, corn starch, lubricating oils, and paints are nonNewtonian fluids having unpredictable viscosity and responses to applied forces. In 1867, Maxwell developed a constitutive model that is used to describe stress relaxation of air. Now, this model has been extensively utilized to analyze the viscoelastic flow characteristics of many industrially important fluids such as liquid polymers, paints, paper pulps, shampoos, flour dough, certain engine oils, and other viscoelastic fluids. On the other hand, flows near stagnation point are commonly encountered in flows around tips of aircrafts, rockets, ships, submarines, etc. These flows also occur in the cooling of electronic devices and nuclear reactors and in many other hydrodynamic processes. The first classical investigation of stagnation point flow of viscous fluids over a flat stationary plate was made by Hiemenz in 1911. He developed an exact solution to the Navier-Stokes equations governing the flow phenomena. The study of fluid flow, heat transfer, and mass diffusion near stagnation point has significantly contributed in understanding and solving problems in manufacturing and engineering. In order to enhance the heat transfer capabilities of traditional fluids, Choi and Eastman [1] introduced the concept of nanofluids in 1995, and they showed experimentally that embedding of nanometer-sized particles with the common base fluids such as water, oil, and ethylene glycol mixture radically increased the thermal conductivity of the fluid. Due to their wide range of applications, a number of experimental and theoretical investigations have been conducted to examine the flow properties of nanofluids in a variety of flow situations. For instance, the heat transfer phenomena of Nanofluid moving over a wedge surface are presented by Ibrahim and Tulu [2]. 
Recently, Zhang et al. [3] analyzed the three-dimensional flow of nanofluid among rotating circular plates. Arain et al. [4] deal with the boundary layer flow characteristics of Carreau fluid in the presence of nanoparticles. Further, Alwawi et al. [5] analyzed the effects of magnetic field and nanoparticles in boundary flow of Casson nanofluids.

A number of researchers have been interested in conducting several investigations on stagnation point flow of Maxwell nanofluids. For instance, Nagendramma et al. [6] considered a two-dimensional stagnation point flow of Maxwell nanofluid over a stretching surface in the presence of thermal radiation and viscous dissipation. Ramesh et al. [7] carried out a numerical study to analyze and discuss a stagnation point flow of a Maxwell nanofluid towards a permeable stretching sheet. Bai et al. [8] investigated a two-dimensional stagnation point flow of Maxwell nanofluid over a stretching surface by taking both the convective heat transfer and the Brownian motion into account. Haritha et al. [9] investigated the combined effect of Navier slip and convective boundary conditions on an unsteady two-dimensional flow of a Maxwell fluid over a stretching surface in the presence of magnetic field, thermal radiation, heat source, and chemical reaction. Mushtaq et al. [10] studied Buoyancy effects in stagnation point flow of Maxwell fluid utilizing non-Fourier heat flux approach. Recently, Khan et al. [11] investigated the heat and mass diffusion effects in the stagnation point flow of Maxwell nanofluid over a stretchable sheet with slip conditions and chemical reaction. Aziz and Shams [12] studied the heat transfer processes due to the flow of electrically conducting Maxwell nanofluid in the context of volumetric entropy generation.

In the aforementioned papers, and in many other early works, the effect of induced magnetic field is usually neglected by assuming an extremely small magnetic Reynolds number and/or getting the mathematical analysis of the problem simple. However, in various practical situations such as power generation, manufacturing of glass, purification of crude oil, and geophysics, the induced magnetic field effect is significant. To this end, extremely few studies are available in open literature that examine the effect of induced magnetic field in stagnation point flow of Maxwell nanofluids. Ibrahim [13] considered the effect of induced magnetic field in examining a stagnation point flow and heat transfer due to upper-convected Maxwell nanofluid over a stretching sheet. In his numerical study, the skin friction coefficient, local Nusselt number, and Sherwood number are found to be decreasing with the increase in the stretching parameter and magnetic field strength. His study also showed that the increase in the convective parameter causes a decrease in the local Sherwood number but an increase in the local Nusselt number. The review of related literature reveals that even though several investigations have been made in the area under consideration, the field remains an active area of research with a number of study gaps. Thus, motivated by the aforementioned early works, this study attempts to examine the influences of magnetic field, thermal radiation, heat source, viscous dissipation, Joule heating, chemical reaction, permeability, and porosity parameters on stagnation point flow of Maxwell nanofluid towards a vertical stretching sheet. Further, a relatively recent and more reliable optimal homotopy analysis method has been implemented successfully to solve the resulting model equations. The results of the present study are also in close agreement with previous works under some restricted assumptions.

\section{Mathematical Formulation of the Flow System}

In this study, it is assumed that as a steady laminar flow of Maxwell nanofluid is approaching a convectively heated vertical sheet, the flow divides into two streams keeping the stagnation point fixed at the origin. Also, a uniform magnetic field $\mathbf{B}$ of strength $B_{0}$ is applied normally to the vertical sheet. Magnetic Reynolds number is taken to be large enough to consider the effect of induced magnetic field. Further, an incompressible, electrically conducting, thermally radiative and chemically reactive fluid is considered, and the permeable sheet is stretching with speed $U_{w}=a x$, where $a>0$ is a constant. It is also assumed that the flow system is embedded in a porous medium as illustrated in Figure 1.

Accordingly, the equations governing the aforementioned flow system can be extended from Ibrahim [13], Hayat and Nadeem [14] as

$$
\begin{aligned}
\frac{\partial u}{\partial x}+\frac{\partial v}{\partial y}= & 0 \\
\frac{\partial H_{1}}{\partial x}+\frac{\partial H_{2}}{\partial y}= & 0, \\
u \frac{\partial u}{\partial x}+v \frac{\partial u}{\partial y}-\frac{\mu_{m}}{\rho_{f}}\left(H_{1} \frac{\partial H_{1}}{\partial x}+H_{2} \frac{\partial H_{1}}{\partial y}-H_{e} \frac{\mathrm{d} H_{e}}{\mathrm{~d} x}\right)= & U_{e} \frac{\partial U_{e}}{\partial x}+v \frac{\partial^{2} u}{\partial y^{2}}-\lambda_{0}\left(u^{2} \frac{\partial^{2} u}{\partial x^{2}}+v^{2} \frac{\partial^{2} u}{\partial y^{2}}+2 u v \frac{\partial^{2} u}{\partial x \partial y}\right) \\
& -\left(\frac{\sigma B_{0}^{2}}{\rho_{f}}+\frac{v}{K_{0}}\right)\left(u-U_{e}\right) \pm \frac{1}{\rho_{f}} g\left[\beta_{0} \rho_{f}\left(1-C_{\infty}\right)\left(T-T_{\infty}\right)\right. \\
& \left.-\left(\rho_{p}-\rho_{\infty}\right)\left(C-C_{\infty}\right)\right],
\end{aligned}
$$




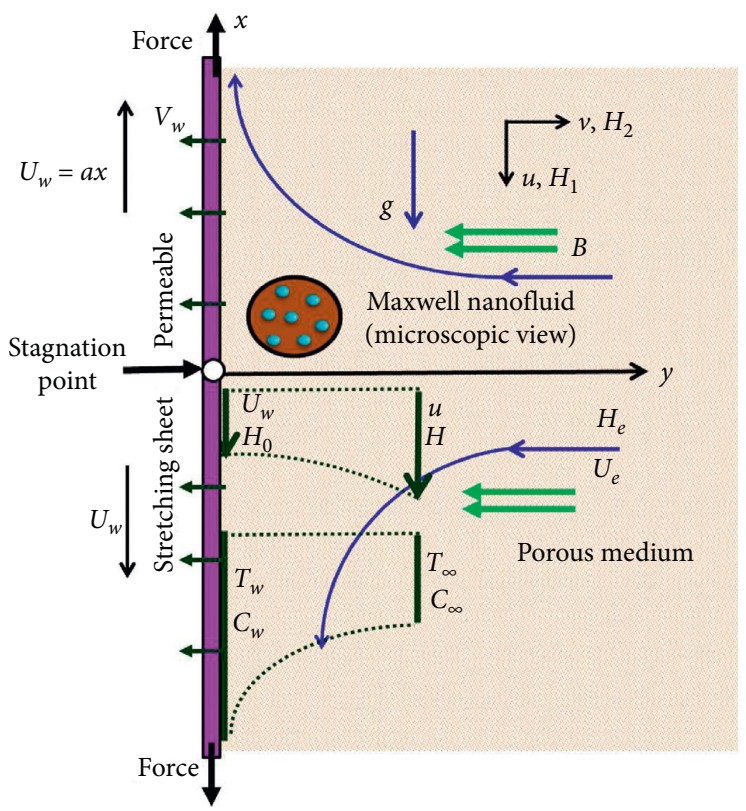

Figure 1: Sketch of the flow problem.

$$
\begin{aligned}
\frac{\partial H_{1}}{\partial t}+u \frac{\partial H_{1}}{\partial x}+v \frac{\partial H_{1}}{\partial y}= & H_{1} \frac{\partial u}{\partial x}+H_{2} \frac{\partial u}{\partial y}+\frac{1}{\sigma \mu_{m}} \frac{\partial^{2} H_{1}}{\partial y^{2}} \\
u \frac{\partial T}{\partial x}+v \frac{\partial T}{\partial y}= & \alpha \frac{\partial^{2} T}{\partial y^{2}}+\frac{v}{C_{p}}\left(\frac{\partial u}{\partial y}\right)^{2}+\frac{1}{\rho C_{p} \sigma}\left(\frac{\partial H_{1}}{\partial y}\right)^{2}+\tau\left[D_{B} \frac{\partial C}{\partial y} \frac{\partial T}{\partial y}+\frac{D_{T}}{T_{\infty}}\left(\frac{\partial T}{\partial y}\right)^{2}\right] \\
& +\frac{\sigma B_{0}^{2}}{\left(\rho C_{p}\right)_{f}}\left(u-U_{e}\right)^{2}+\frac{16 \sigma^{*} T_{\infty}^{3}}{3\left(\rho C_{p}\right)_{f} k^{*}} \frac{\partial^{2} T}{\partial y^{2}}+\frac{Q_{0}}{\left(\rho C_{p}\right)_{f}}\left(T-T_{\infty}\right), \\
u \frac{\partial C}{\partial x}+v \frac{\partial C}{\partial y}= & D_{B} \frac{\partial^{2} C}{\partial y^{2}}+\frac{D_{T}}{T_{\infty}} \frac{\partial^{2} T}{\partial y^{2}}-K_{r}\left(C-C_{\infty}\right),
\end{aligned}
$$

where $(u, v)$ and $\left(H_{1}, H_{2}\right)$ denote the components of fluid velocity vector $\mathbf{V}$ and induced magnetic field vector $\mathbf{H}$ along $x$ and y directions, $\rho_{f}, \mu_{m}, \lambda_{0}, \sigma, \kappa$ and $\beta_{0}$ denote density, magnetic permeability, viscoelasticity, electric conductivity, thermal conductivity, and thermal expansion of the nanofluid, respectively; the quantities $g$ and $K_{0}$ represent the acceleration due to gravity and permeability of the porous medium, respectively; $v=\left(\mu / \rho_{f}\right)$ is kinematic viscosity of the nanofluid with $\mu$ denoting coefficient of dynamic viscosity; $U_{e}$ and $H_{e}$ are the free stream velocity and induced magnetic field; $T$ and $C$ denote temperature and nanoparticle volume fraction; $T_{\infty}$ and $C_{\infty}$ are the corresponding ambient values of temperature and concentration; $\alpha=\left(\kappa /\left(\rho C_{p}\right)_{f}\right)$ is thermal diffusivity, and $\tau=\left(\left(\rho C_{p}\right)_{p} /\right.$ $\left.\left(\rho C_{p}\right)_{f}\right)$ is the ratio of effective heat capacities of nanoparticle and the ordinary fluid; $D_{B}$ and $D_{T}$ are the Brownian and thermophoresis diffusion coefficients; $k^{*}$ and $\sigma^{*}$ are the mean absorption and the Stefan-Boltzmann constants, respectively; the coefficient $Q_{0}$ stands for heat generation (when $Q_{0}>0$ ) and heat absorption (when $Q_{0}<0$ ); the rates $K_{r}>0$ and $K_{r}<0$ denote destructive and constructive reaction rates, respectively.

Taking the boundary conditions,

at $y=0$,

$$
\begin{aligned}
u & =U_{w}(x)=a x, \\
\frac{\partial H_{1}}{\partial y} & =H_{2}=0, \\
v & =V_{w}(t)=-V_{0}, \\
-k \frac{\partial T}{\partial y} & =h_{f}\left[T_{w}-T\right], \\
C & =C_{w},
\end{aligned}
$$

and as $y \longrightarrow \infty$, 


$$
\begin{aligned}
u & \longrightarrow u_{e}(x)=b x, \\
v & \longrightarrow 0 \\
H_{1} & \longrightarrow H_{e}(x)=x H_{0}, \\
T & \longrightarrow T_{\infty}, \\
C & \longrightarrow C_{\infty},
\end{aligned}
$$

where $U_{w}, T_{w}, C_{w}$ denote the surface velocity, temperature, and nanoparticle concentration, respectively; $V_{w}$ is the mass transmission at the surface of the stretching sheet; $V_{0}$ is the constant value of velocity; $k$ and $h_{f}$ are thermal diffusivity and convective heat transfer coefficients, respectively; $H_{0}$ is the initial induced magnetic field strength; $a$ and $b$ are positive constants denoting velocity rate of the stretching sheet and the fluid in the free stream, respectively.

In order to further simplify the mathematical analysis, we use the following transformations:

$$
\begin{aligned}
\eta & =y \sqrt{\frac{a}{v}} \\
\psi(x, y, t) & =\sqrt{a v} x f(\eta), \\
H_{1} & =x H_{0} g^{\prime}(\eta), \\
H_{2} & =-H_{0} \sqrt{\frac{v}{a}} g(\eta), \\
\theta(\eta) & =\frac{T-T_{\infty}}{T_{w}-T_{\infty}}, \\
\varphi(\eta) & =\frac{C-C_{\infty}}{C_{w}-C_{\infty}},
\end{aligned}
$$

where $\eta$ is a dimensionless similarity variable; $f^{\prime}(\eta), g(\eta), \theta(\eta)$ and $\varphi(\eta)$ denote the dimensionless functions for velocity, induced magnetic field, temperature, and concentration profiles.

Introducing the stream function $\psi(x, y)$ with the property $u=(\partial \psi / \partial y)$ and $v=-(\partial \psi / \partial x)$, the continuity equation for velocity in equation (1) is identically satisfied. Also, from the definition of $H_{1}$ and $H_{2}$ in equation (9), the continuity equation for induced magnetic field equation (2) was satisfied identically. Computing the required partial derivatives and substituting the values into the governing equations (3)-(6), the following system of nonlinear differential equations can be obtained:

$$
\left\{\begin{array}{l}
f^{\prime \prime \prime}-f f^{\prime \prime}-M\left(g^{\prime 2}-g g^{\prime \prime}-1\right)+\lambda\left(2 f f^{\prime} f^{\prime \prime}-f^{2} f^{\prime \prime \prime}\right)+\left(H_{a}^{2}+K_{p}\right)\left(\delta-f^{\prime}\right)+r\left(\theta-N_{r} \varphi\right)+\delta^{2}=0 \\
\varepsilon g^{\prime \prime \prime}+g^{\prime \prime} f-g f^{\prime \prime}=0 \\
\frac{1}{P_{r}}\left(1+\frac{4}{3} R_{d}\right) \theta^{\prime \prime}+f \theta^{\prime}+N_{b} \theta^{\prime} \varphi^{\prime}+N_{t} \theta^{\prime 2}+E_{c}\left[f^{\prime \prime 2}+H_{a}^{2}\left(f^{\prime 2}-2 \delta f^{\prime}+\delta^{2}\right)\right]+Q \theta=0 \\
\varphi^{\prime \prime}+\frac{N_{t}}{N_{b}} \theta^{\prime \prime}+S_{c}\left[f \varphi^{\prime}-\gamma \varphi\right]=0
\end{array}\right.
$$

where the prime indicates differentiation with respect to $\eta$; $M=\left(\mu_{m} H_{0}^{2} / \rho v^{2}\right)$ and $H_{a}^{2}=\left(\sigma B_{0}^{2} / a \rho_{f}\right)\left(u-U_{e}\right)$ are parameters representing the induced and the external magnetic field strengths, respectively; $\lambda=a \lambda_{0} \geq 0$ is the Deborah number representing the Maxwell viscoelastic parameter; $K_{p}=\left(v x / U_{w} K_{0}\right)$ is the porosity parameter; $\delta=(b / a)$ is a velocity ratio representing the sheet stretching rate, with $\delta \geq 1$ and $\delta<1$, respectively, implying that the fluid free stream velocity is greater than the sheet velocity and vice versa; $r= \pm\left(\beta_{0} g\left(T_{w}-T_{\infty}\right)\left(1-C_{\infty}\right) / a^{2} x\right)$ is a gravity dependent parameter for assisting (when $r>0$ ) and opposing (when $r<0)$ flows; $N_{r}=\left(\left(\rho_{p}-\rho_{\infty}\right)\left(C_{w}-C_{\infty}\right) /\right.$ $\left.\rho_{f} \beta_{0}\left(T_{w}-T_{\infty}\right)\left(1-C_{\infty}\right)\right)$ is the buoyancy ratio; $\varepsilon=$ $\left(1 / \mu_{m} \sigma v\right)>0$ is the magnetic diffusivity number; $P_{r}=(v / \alpha)$ and $S_{c}=\left(v / D_{B}\right)$ are the Prandtl number and the Schmidt number, respectively; $R_{d}=\left(4 \sigma^{*} T_{\infty}^{3} /\left(\rho C_{p}\right){ }_{f} k^{*}\right)$ is the thermal radiation parameter; $E_{c}=\left(U_{w}^{2} /\left(C_{p}\right)_{f}\left(T_{w}-T_{\infty}\right)\right)$ is the Eckert number that represents dissipation parameter; $N_{b}=$ 
$\left(\tau D_{B}\left(C_{w}-C_{\infty}\right) / v\right)$ and $N_{t}=\left(\tau D_{T}\left(T_{w}-T_{\infty}\right) / v T_{\infty}\right)$ are the nanofluid parameters that denote Brownian motion, which represent mass transfer and thermophoresis effects, which represent heat transfer characteristics, respectively; $Q=\left(x Q_{0} /\left(\rho C_{p}\right)_{f} U_{w}\right)$ is the heat generation (for $\left.Q>0\right)$ or absorption (for $Q<0)$ parameter; and $\gamma=\left(K_{r} x / U_{w}\right)$ is the chemical reaction parameter, with $\gamma>0$ and $\gamma<0$ indicating the destructive and generative chemical reaction rates, respectively. Also employing the transformation in equation (9), the boundary conditions in equations (7) and (8) can be reduced as follows:

$$
\left\{\begin{array}{l}
f(0)=S, \\
f^{\prime}(0)=1, \\
g(0)=g^{\prime \prime}(0)=0, \\
\theta^{\prime}(0)=-\operatorname{Bi}[1-\theta(0)], \\
\varphi(0)=1, \\
f^{\prime}(\eta) \longrightarrow \delta, \\
g^{\prime}(\eta) \longrightarrow 1, \\
\theta(\eta) \longrightarrow 0, \\
\varphi(\eta) \longrightarrow 0, \quad \text { as } \eta \longrightarrow \infty,
\end{array}\right.
$$

where the parameter $S=\left(V_{0} / \sqrt{a v}\right)$ denotes injection and suction for $S>0$ and $S<0$, respectively; $B_{i}=\left(h_{f} / k\right) \sqrt{v / a}$ is the Biot number representing the convective parameter.

From a practical point of view, we exploit the behavior of Skin friction $C_{f}$, local Nusselt number $\mathrm{Nu}_{x}$, and Sherwood number $\mathrm{Sh}_{x}$ in the region of stagnation point defined, respectively, as

$$
\begin{aligned}
& C f_{x}=\frac{2 \tau_{w}}{\rho_{f} U_{w}^{2}}, \\
& \mathrm{Nu}_{x}=\frac{x q_{w}}{\kappa\left(T_{w}-T_{\infty}\right)}, \\
& \mathrm{Sh}_{x}=\frac{x J_{w}}{D_{B}\left(C_{w}-C_{\infty}\right)},
\end{aligned}
$$

where $\tau_{w}=\mu_{0}(1+\lambda)[\partial u / \partial y]_{y=0}$ is the shear stress; $q_{w}=-\left(\kappa+\left(16 \sigma * T_{\infty}^{3} / 3\left(\rho C_{p}\right)_{f} k^{*}\right)\right)[\partial T / \partial y]_{y=0}$ is the heat flux; and $J_{w}=-D_{B}[\partial C / \partial y]_{y=0}$ is the mass flux at the stretching surface.

Upon substitution, this gives

$$
\begin{aligned}
\operatorname{Re}_{x}^{1 / 2} C f_{x} & =2(1+\lambda) f^{\prime \prime}(0), \\
\mathrm{Nu}_{x} & =-\left(1+\frac{4}{3} R_{d}\right) \operatorname{Re}_{x}^{1 / 2} \theta^{\prime}(0), \\
\operatorname{Re}_{x}^{-(1 / 2)} \operatorname{Sh}_{x} & =-\varphi^{\prime}(0)
\end{aligned}
$$

where $\operatorname{Re}_{x}=\left(x U_{w} / v\right)=\left(a x^{2} / v\right)$ is the local Reynolds number.

\section{Method of Solution}

The homotopy analysis method (HAM) is one of the relatively recent mathematical methods showing greater efficiency over the past few years. The method is also known for its flexibility and high accuracy to give analytic approximations to the solutions of many important problems in science and engineering. Details of the method can be found in Liao $[15,16]$. In order to implement the homotopy analysis method, we first choose the following auxiliary linear operators:

$$
\begin{aligned}
& L_{f}(f)=\frac{\mathrm{d}^{3} f}{\mathrm{~d} \eta^{3}}+\frac{\mathrm{d}^{2} f}{\mathrm{~d} \eta^{2}}, \\
& L_{g}(g)=\frac{\mathrm{d}^{3} g}{\mathrm{~d} \eta^{3}}+\frac{\mathrm{d}^{2} g}{\mathrm{~d} \eta^{2}}, \\
& L_{\theta}(\theta)=\frac{\mathrm{d}^{2} \theta}{\mathrm{d} \eta^{2}}+\frac{\mathrm{d} \theta}{\mathrm{d} \eta} \\
& L_{\varphi}(\varphi)=\frac{\mathrm{d}^{2} \varphi}{\mathrm{d} \eta^{2}}+\frac{\mathrm{d} \varphi}{\mathrm{d} \eta}
\end{aligned}
$$

satisfying the conditions

$$
\begin{aligned}
L_{f}\left[C_{1}+C_{2} \eta+C_{3} e^{-\eta}\right] & =0, \\
L_{g}\left[C_{4}+C_{5} \eta+C_{6} e^{-\eta}\right] & =0, \\
L_{\theta}\left[C_{7}+C_{8} e^{-\eta}\right] & =0, \\
L_{\varphi}\left[C_{9}+C_{10} e^{-\eta}\right] & =0,
\end{aligned}
$$

where $C_{i}(i=1-10)$ are integral constants to be determined by the boundary conditions. Next, based on the linear operators and the boundary conditions, we take the following initial approximations:

$$
\begin{aligned}
& f_{0}(\eta)=S+\delta \eta+(1-\delta)\left(1-e^{-\eta}\right) \\
& g_{0}(\eta)=\eta \\
& \theta_{0}(\eta)=\frac{\mathrm{Bi}}{1+\mathrm{Bi}} e^{-\eta} \\
& \varphi_{0}(\eta)=e^{-\eta}
\end{aligned}
$$

The nonzero auxiliary functions can also be selected as

$$
H_{f}(\eta)=H_{g}(\eta)=H_{\theta}(\eta)=H_{\varphi}(\eta)=e^{-\eta} \text {. }
$$

Finally, the parameter values $\delta=\varepsilon=1.5, P_{r}=S_{c}=1$, $H_{a}=0.04, N_{b}=N_{r}=E_{c}=r=0.1, N_{c}=B_{i}=0.5, M=\lambda=$ $K_{p}=N_{t}=R_{d}=Q=S=0.2$, and $\gamma=0.1$ are used throughout this study unless otherwise stated. In order to do the required computations, we employ a powerful computational software, namely, the BVPh2.0, which is a HAM-based Mathematica package, developed by Zhao and Liao [17].

Now, before presenting the results of the study, we give the following convergence analysis of the method. Since convergence of the HAM solutions depends strongly on the 
proper choice of the so-called convergence-control parameters, we determine the admissible values of $\hbar_{f}, \hbar_{g}, \hbar_{\theta}$ and $\hbar_{\varphi}$ as follows. According to Liao [15], one of the mechanisms to identify the range of such values is to sketch the $\hbar$-curves as shown in Figure 2. This result illustrates that the $\hbar$-curves are nearly horizontal in the intervals:

$$
\begin{aligned}
& -1.7<\hbar_{f}<-0.2, \\
& -1.6<\hbar_{g}<0.2, \\
& -1.7<\hbar_{\theta}<-0.1, \\
& -1.6<\hbar_{\varphi}<-0.3 .
\end{aligned}
$$

So, any value in these intervals may be taken to get convergent solutions to our flow problem.

More systematically, the optimal values for the convergence-control parameters are determined by reducing the squared residual error:

$$
\varepsilon_{k}(\hbar) \approx \frac{1}{1+N} \sum_{j=0}^{N}\left\{\aleph\left[\sum_{n=0}^{k} u_{n}\left(\eta_{j}\right)\right]\right\}^{2} .
$$

Upon using the BVPh2.0 Mathematica package, it has been found that

$$
\begin{aligned}
& \hbar_{f} \approx-1.4565, \\
& \hbar_{g} \approx-1.2499, \\
& \hbar_{\theta} \approx-0.8458, \\
& \hbar_{\varphi} \approx-1.1501 .
\end{aligned}
$$

Further, the respective residual errors and the required values of derivatives are presented against the orders of HAM approximations in Table 1.

It is clear from Table 1 and Figure 3 that increasing the order of HAM approximation decreases the squared residual errors and leads the series to converge.

The sketch in Figure 3 clearly indicated that the total error is decaying extremely fast for increasing orders of the first few iterations. This guarantees that the method is convergent for the selected optimal values of the convergence parameters. To further ensure the validity of our work, we made a comparison of our results with respect to some other previous reports in the absence of extended physical effects as presented in Table 2 .

Table 2 verifies that, for the selected values of the parameters, the values of the physical quantities of practical interest determined in this study are in excellent agreement with those of the results reported by Hayat and Nadeem [14].

\section{Results and Discussions}

In this section, we present the most significant results of the study in graphical or tabular forms followed by brief discussions. The impacts of various thermophysical parameters on the dimensionless velocity $f^{\prime}(\eta)$, induced magnetic field $g(\eta)$, temperature $\theta(\eta)$, and concentration $\varphi(\eta)$ profiles in the stagnation point region are presented as follows:

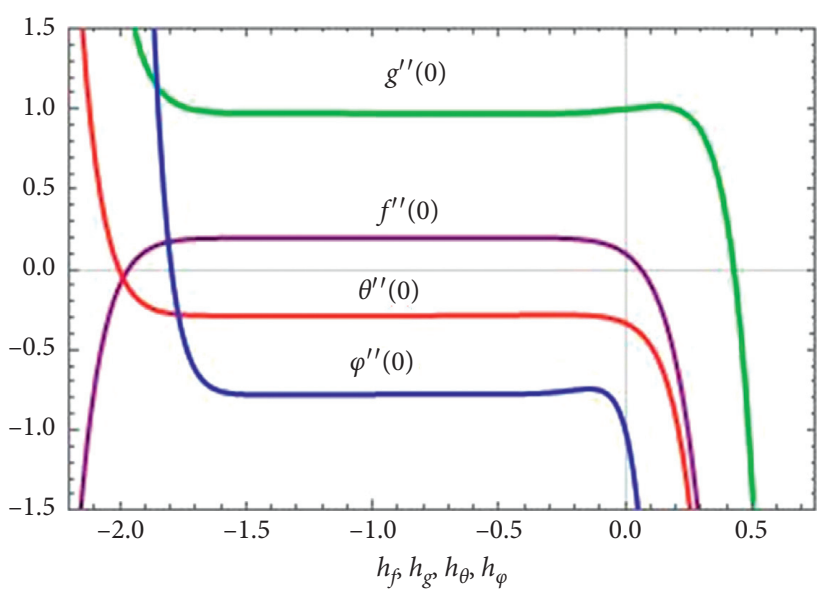

Figure 2: The $\hbar$-curves at $15^{\text {th }}$ order HAM approximations.

The effects of external magnetic field in the flow field profiles can be expressed in terms of the Hartman number $\mathrm{Ha}$ as presented in Figure 4.

Here, it is worth mentioning that the term $\left(\sigma B_{0}^{2} / \rho\right)(u-$ $U_{e}$ ) can be viewed as the combination of the imposed pressure term $\left(\left(\sigma B_{0}^{2} / \rho\right) U_{e}\right)$ and the Lorentz force $\left(\left(\sigma B_{0}^{2} / \rho\right) u\right)$. Unlike free convection, for forced convection regime, the external flow velocity $U_{e}$ is greater than the velocity $u$ of the fluid in the boundary layer region. That is, the imposed pressure overcomes the Lorentz force. This corresponds to an assisting force in the direction of main flow. Thus, the increase in external magnetic field advances the flow velocity and the induced magnetic field. However, the opposite behavior is observed for temperature profile.

The influence of stretching parameter $\delta$ on the dimensionless profiles in the region of stagnation point has been investigated and illustrated graphically in Figures 5(a) and 5(b). It is revealed that as the parameter $\delta$ increases, the velocity profile also increases for both $\delta<1$ and $\delta \geq 1$ but with inverted boundary layer structures. This is due to the fact that as the velocity ratio parameter increases, the velocity of the straining motion near the stretching surface also increases from the smooth intersection of the external fluid and the sheet. Also, the induced magnetic field profile was found to be increasing for $\delta<1$ and decreasing for $\delta \geq 1$. On the other hand, temperature and concentration profiles have remained decreasing as we move away from the stretching sheet.

The influences of buoyancy ratio parameter $N_{r}$ on dimensionless velocity, induced magnetic field, temperature, and concentration profiles for both assistive and resistive flows are analyzed and given in Figures 6(a) and 6(b).

For resistive flow $r<0$, it can be observed from Figure 6(a) that both the velocity and induced magnetic field profiles are increasing functions of $N_{r}$. On the other hand, temperature and concentration profiles slowly decrease with $N_{r}$. The opposite behavior of the profiles can be noticed for the assistive flow $r>0$ as shown in Figure 6(b). This is true because, in the assistive flow, the buoyancy force acts against the flow field. 
TABle 1: Convergence of some HAM solutions for $\delta=\gamma=r=N_{r}=E_{c}=0.1, P_{r}=S_{c}=1, \varepsilon=1.5, B_{i}=0.5, \lambda=N_{b}=N_{t}=K_{p}=M=$ $R_{d}=Q=S=0.2, H_{a}=0.04$.

\begin{tabular}{|c|c|c|c|c|c|c|c|}
\hline \multirow{2}{*}{ Order of approx. } & \multirow{2}{*}{$f^{\prime \prime}(0)$} & \multirow{2}{*}{$-\theta^{\prime}(0)$} & \multirow{2}{*}{$-\varphi^{\prime}(0)$} & \multicolumn{4}{|c|}{ Squared residual errors } \\
\hline & & & & $\varepsilon_{f}$ & $\varepsilon_{g}$ & $\varepsilon_{\theta}$ & $\varepsilon_{\varphi}$ \\
\hline 2 & 0.1988 & 0.2832 & 0.7457 & $7.06 \times 10^{-6}$ & $5.38 \times 10^{-6}$ & $9.40 \times 10^{-5}$ & $2.82 \times 10^{-3}$ \\
\hline 6 & 0.1966 & 0.2823 & 0.7812 & $2.19 \times 10^{-8}$ & $6.59 \times 10^{-7}$ & $2.12 \times 10^{-5}$ & $3.08 \times 10^{-5}$ \\
\hline 10 & 0.1965 & 0.2858 & 0.7800 & $4.94 \times 10^{-9}$ & $8.65 \times 10^{-9}$ & $4.09 \times 10^{-6}$ & $1.23 \times 10^{-5}$ \\
\hline 14 & 0.1964 & 0.2876 & 0.7792 & $3.65 \times 10^{-9}$ & $1.06 \times 10^{-8}$ & $9.81 \times 10^{-7}$ & $4.90 \times 10^{-6}$ \\
\hline 18 & 0.1964 & 0.2883 & 0.7797 & $1.79 \times 10^{-9}$ & $2.25 \times 10^{-9}$ & $4.44 \times 10^{-7}$ & $2.08 \times 10^{-6}$ \\
\hline 22 & 0.1964 & 0.2886 & 0.7803 & $8.24 \times 10^{-10}$ & $9.91 \times 10^{-10}$ & $2.90 \times 10^{-7}$ & $1.15 \times 10^{-6}$ \\
\hline 26 & 0.1964 & 0.2886 & 0.7808 & $3.97 \times 10^{-10}$ & $4.62 \times 10^{-10}$ & $1.93 \times 10^{-7}$ & $7.92 \times 10^{-7}$ \\
\hline 30 & 0.1964 & 0.2885 & 0.7811 & $2.07 \times 10^{-10}$ & $1.96 \times 10^{-10}$ & $1.21 \times 10^{-7}$ & $5.79 \times 10^{-7}$ \\
\hline
\end{tabular}

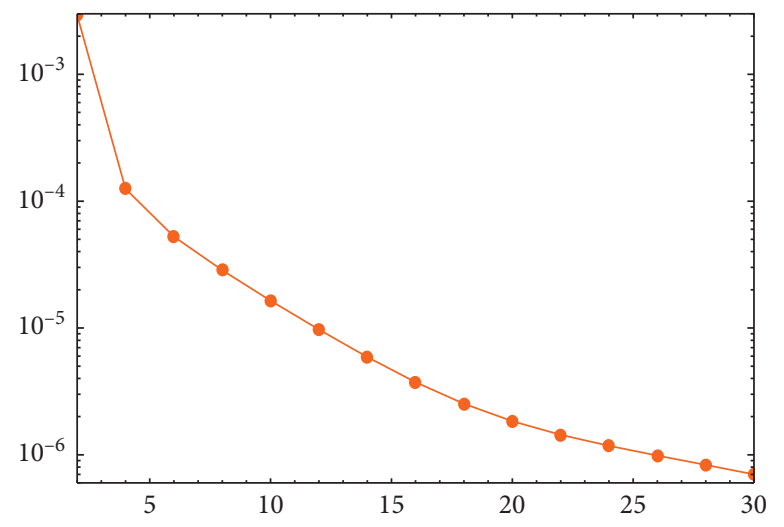

FIgURE 3: Total squared residual error for the $20^{\text {th }}$ HAM approximation.

TABLE 2: Comparisons of the present study with previously published work on the coefficients $f^{\prime \prime}(0),-\theta(0)$, and $-\varphi(0)$ when $\varepsilon=1.5, B_{i}=0.5, \delta=1.1, P_{r}=S_{c}=1, E_{c}=r=N_{r}=N_{b}=N_{t}=0.1, \lambda=H_{a}=K_{p}=R_{d}=Q=\gamma=S=0$ against some values of $M$.

\begin{tabular}{|c|c|c|c|c|c|c|}
\hline \multirow{2}{*}{$M$} & \multicolumn{2}{|c|}{$C_{f} R_{e}^{1 / 2}$} & \multicolumn{2}{|c|}{$N_{U x} R_{e}^{-(1 / 2)}$} & \multicolumn{2}{|c|}{$S_{h x} R_{e}^{-(1 / 2)}$} \\
\hline & {$[14]$} & Present study & [14] & Present study & {$[14]$} & Present study \\
\hline 0.0 & 0.168376 & 0.1683288 & 0.158817 & 0.1588010 & 0.744361 & 0.7448927 \\
\hline 0.2 & 0.159333 & 0.1594507 & 0.158795 & 0.1587788 & 0.743906 & 0.7439417 \\
\hline 0.5 & 0.143776 & 0.1436547 & 0.158730 & 0.1587352 & 0.741125 & 0.7421857 \\
\hline
\end{tabular}

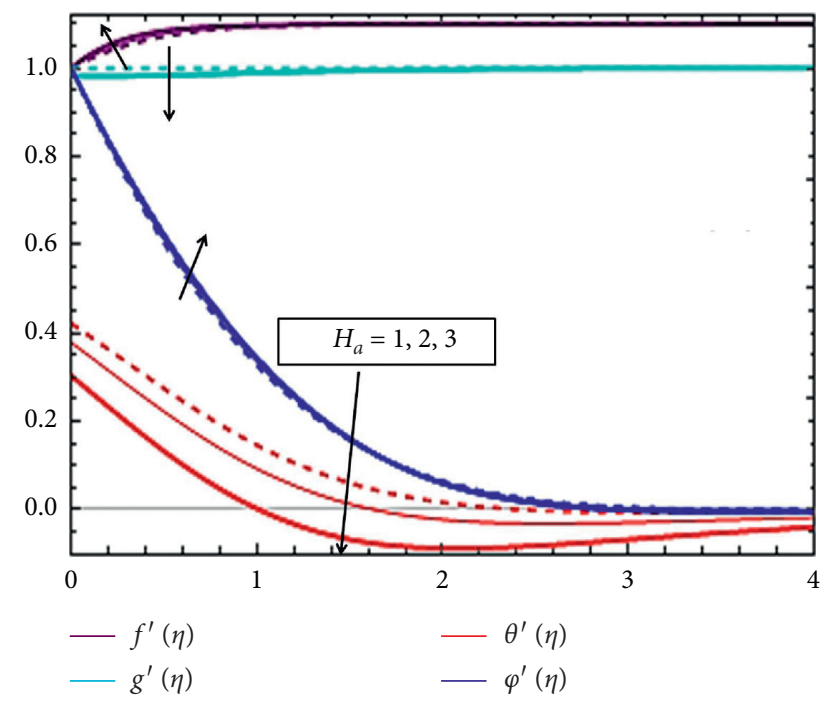

Figure 4: Impacts of Hartman number on velocity, induced magnetic field, temperature, and concentration profiles. 


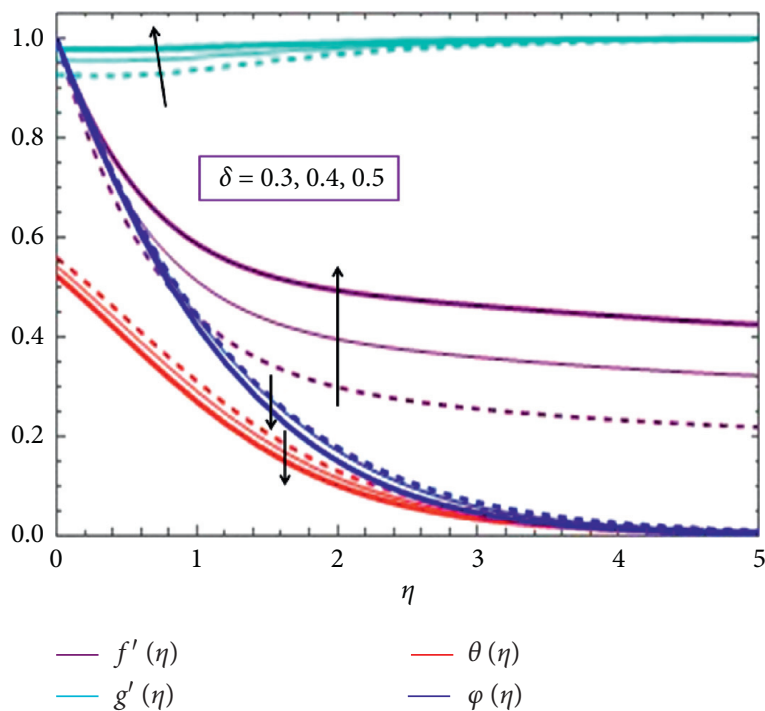

(a)

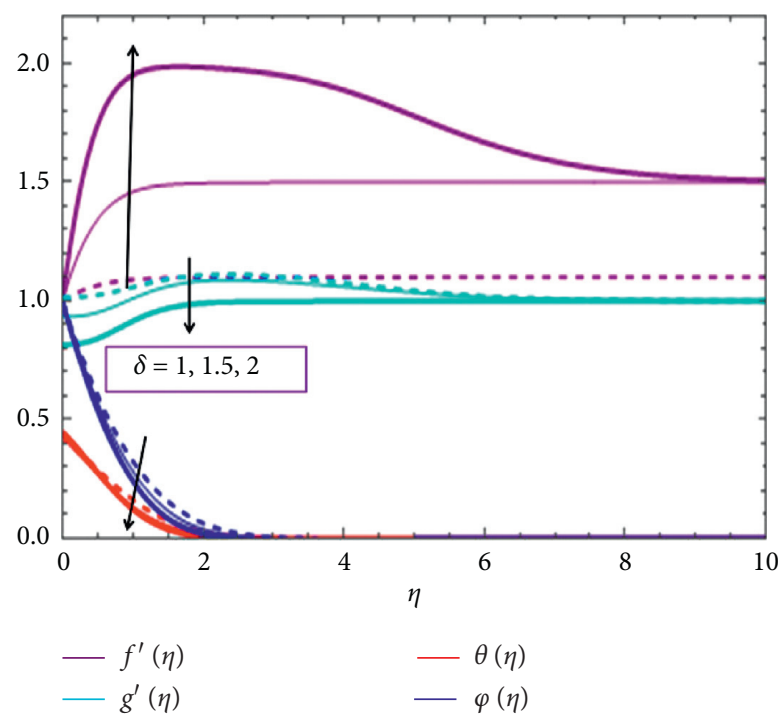

(b)

Figure 5: (a) Impacts of $\delta$ on $f^{\prime}(\eta), g^{\prime}(\eta), \theta(\eta)$, and $\varphi(\eta)$ for $\delta<1$. (b) Impacts of $\delta$ on $f^{\prime}(\eta), g^{\prime}(\eta), \theta(\eta)$, and $\varphi(\eta)$ for $\delta \geq 1$ (right) of the sheet.

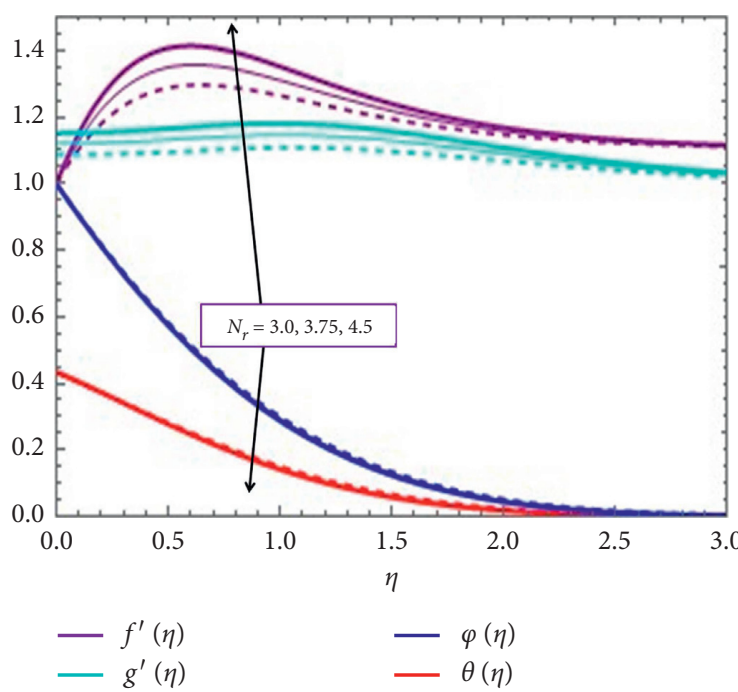

(a)

FiguRE 6: (a) Effects of $N_{r}$ for resistive $(r<0)$ flow

The effect of Maxwell viscoelastic parameter expressed in terms of the Deborah number $\lambda$ has been investigated and presented in Figures 7(a) and 7(b).

As pointed out in Figures $7(\mathrm{a})$ and $7(\mathrm{~b})$, the velocity profile is increasing function of $\lambda$. Physically, at higher Deborah numbers, elasticity of the material increases, while viscosity decreases, which enhances the fluid velocity. Similarly, the induced magnetic field also increases with the increase in the values of $\lambda$. On the other hand, the temperature gradually decreases, and no significant fluctuation of concentration is observed with increase in the values of $\lambda$.

The impact of the convective parameter $B_{i}$ has been studied and illustrated in Figure 8. It can be noticed that the

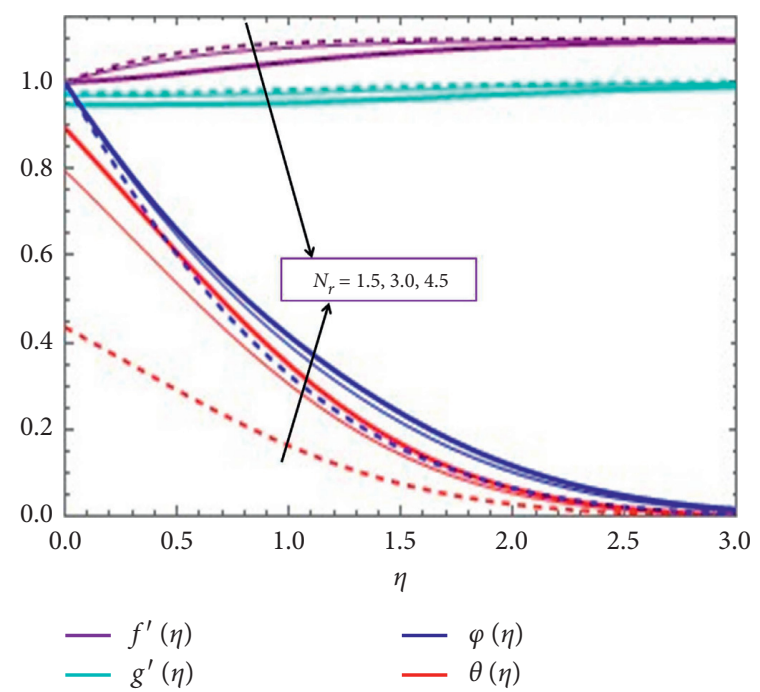

(b) velocity, temperature, and concentration profiles can be lowered by increasing the Biot number.

It can be deduced from Figure 8 that the influence of $B_{i}$ is more prominent on temperature profile near the vertical sheet. It is shown that the temperature profile is declining for increasing values of $B_{i}$.

The reciprocal of magnetic Prandtl number $\varepsilon$ is the ratio of the magnetic diffusivity to viscous diffusivity. Its impact in the flow field profiles has been examined and shown in Figures 9(a) and 9(b).

As shown in Figures 9(a) and 9(b), the increase in the reciprocal of magnetic Prandtl number $\varepsilon$ causes the increase in momentum and induced magnetic field profiles near the 


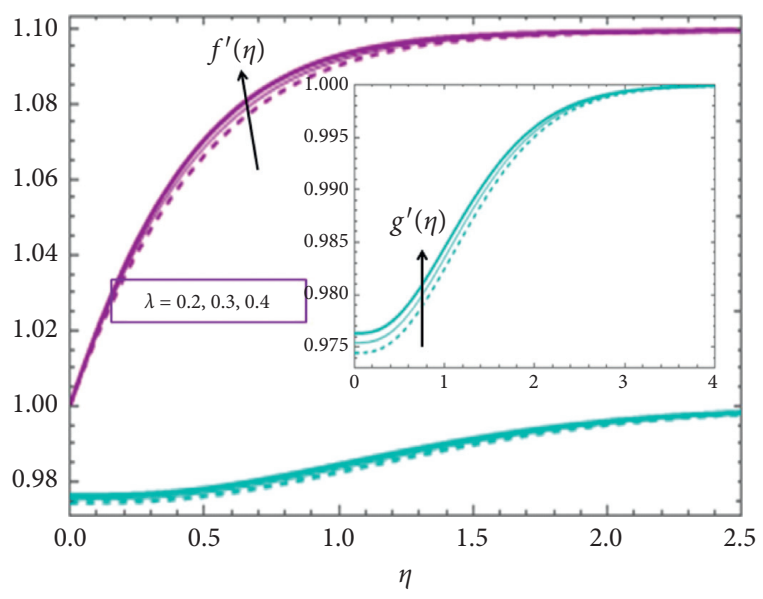

(a)

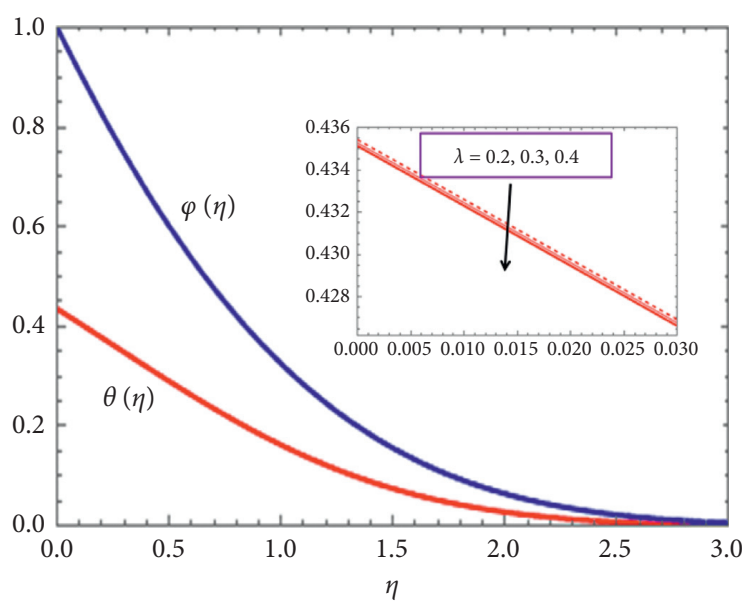

(b)

Figure 7: (a) Effects of $\lambda$ on velocity and induced magnetic field profiles. (b) Effects of $\lambda$ on temperature and concentration profiles.

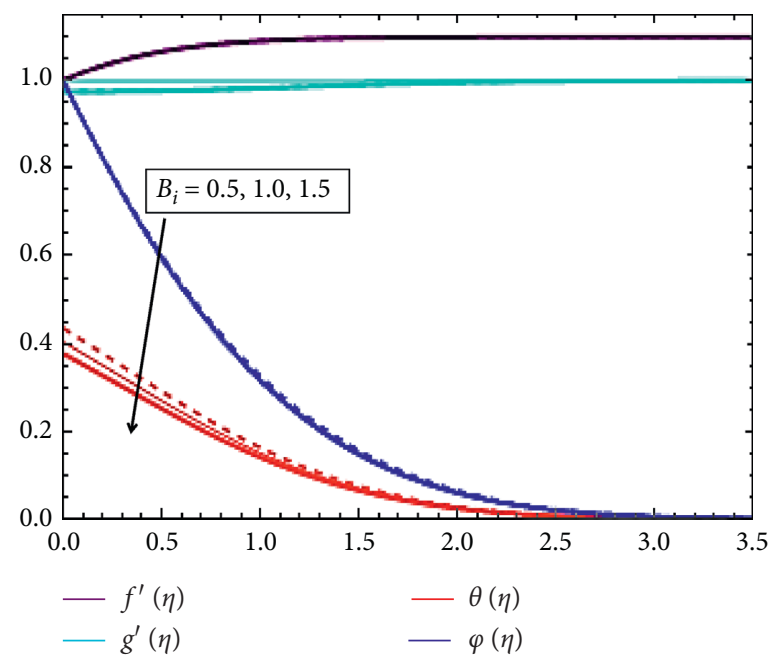

FIGURE 8: Impacts of Biot number on velocity, induced magnetic field, temperature, and concentration profiles.

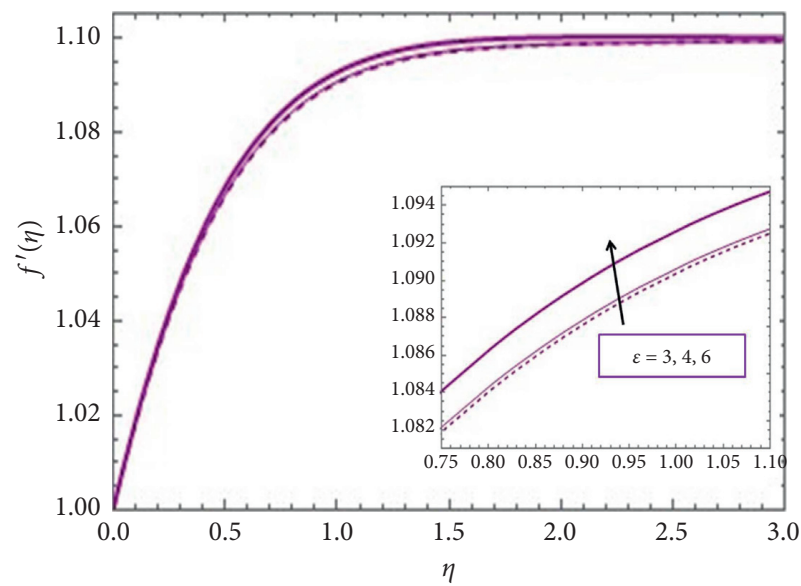

(a)

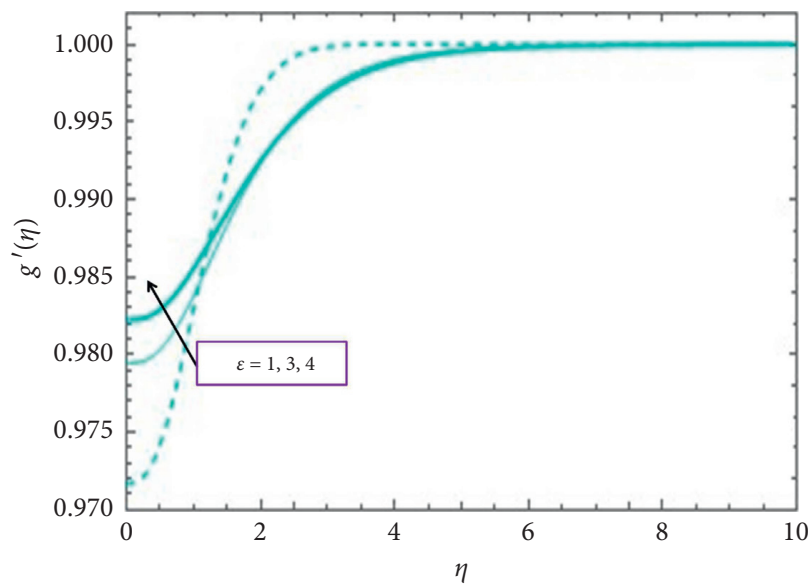

(b)

Figure 9: (a) Effects of $\varepsilon$ on velocity profile. (b) Effects of $\varepsilon$ on induced magnetic field profile. 


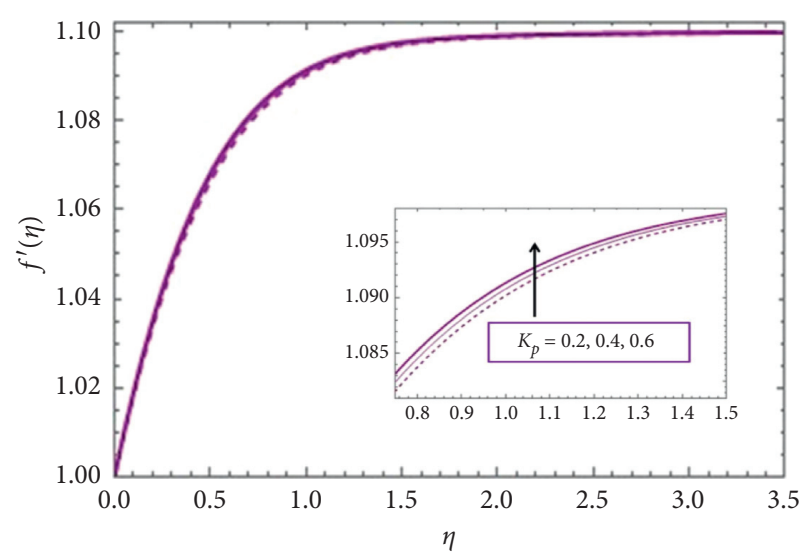

(a)

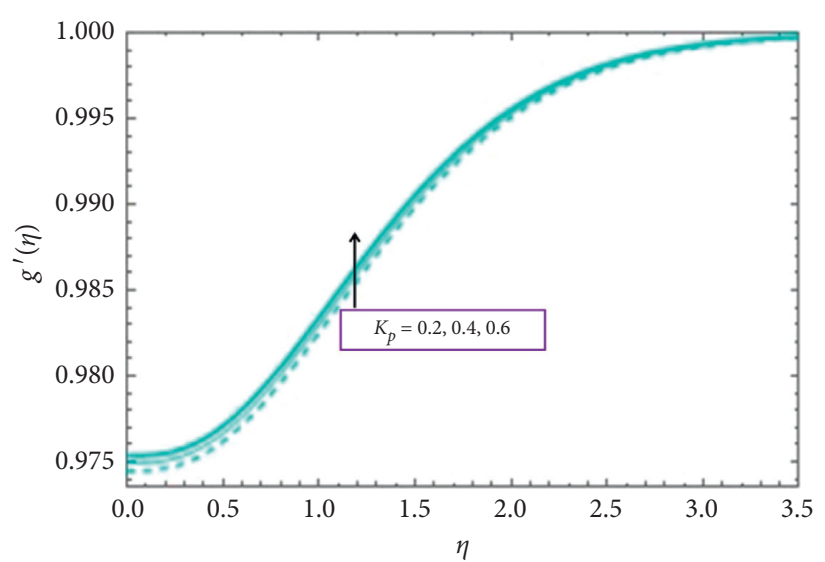

(b)

FIGURE 10: (a) Impacts of porosity parameter $K_{p}$ on velocity profile. (b) Impacts of porosity parameter $K_{p}$ on induced magnetic field profile.

stretching surface. This is evident from the relation that the reciprocal of magnetic Prandtl number is inversely proportional to momentum diffusivity, which is low near the stretching sheet.

Similarly, the effect of porosity in the flow problem has been investigated and presented as in Figures 10(a) and 10(b).

It can be observed in Figures 10(a) and 10(b) that the velocity and induced magnetic field profiles increase as porosity of the medium increases in the flow field.

The influence of radiation $R_{d}$ in the region of stagnation point flow has been determined as depicted in Figure 11(a) and $11(b)$.

The results in Figures 11(a) and 11(b) show that the increment in thermal radiation leads to the growth of thermal boundary layer. Physically, this is due to the fact that larger thermal radiation develops higher heat flux at the surface. It is also noted that as thermal radiation increases, the velocity and induced magnetic field profiles also increase, but the nanoparticle concentration profile reduces in the stagnation point region.

Chemical reactions occur in many industrial and engineering activities such as hydrolysis, electroplating, and combustion processes.

According to the results in Figures 12(a) and 12(b), as the destructive chemical reaction $(\gamma>0)$ increases, the induced magnetic field profile has been increased near the stretching sheet, and the concentration profile decreases in the region of stagnation point. On the other hand, the concentration profile increases with the constructive chemical reaction $(\gamma<0)$ causing the raise of nanoparticle concentration at the sheet. This holds as constructive chemical reactions $(\gamma<0)$ result in consumption, while a destructive chemical reaction $(\gamma>0)$ causes production of the chemical species.

In many situations, heat is generated within a body by a chemical, electrical, or nuclear process. The heat source parameter $Q$ has been used to analyze the effects of heat generation and heat absorption as shown in Figures 13(a)-13(d).
As presented in Figures 13(a) and 13(b), the increase in heat generation results in the increasing of velocity and induced magnetic field profiles. Figures 13(c) and 13(d) show that the temperature of the nanofluid increases with heat generation and decreases with heat absorption. Here, the effect of heat generation yields thermal energy; thereby, the temperature rises, while heat absorption causes the removal of thermal energy in which the temperature profile declines.

Viscous dissipation is the conversion of kinetic energy into thermal energy due to the work done against the viscous fluid. The effects of viscous dissipation can be demonstrated by using the Eckert number Ec.

It is shown in Figure 14(a) that the increases in the dissipation parameter cause the increase in temperature profile. Physically, this is true because the increase in the dissipation effect implies a rise in the frictional heating, which leads to storing of more heat energy in the flow system. Also, Figures 14(b) and 14(c) display that the velocity and induced magnetic field profiles are found to increase with the increase in the dissipation parameter.

This study also examines the permeability effects in terms of the suction/injection parameter $S$ as depicted in Figures 15(a) and 15(b).

It can be seen from Figures 15(a) and 15(b) that both the velocity and induced magnetic field profiles are increasing functions of the injection parameter. On the other hand, the temperature and concentration profiles both decrease with the increase in the injection parameter.

The impacts of some parameters on the rates of momentum, heat, and mass transfer near the stretching sheet are examined and expressed in terms of the skin friction, local Nusselt number, and Sherwood number. To this end, the values of the corresponding boundary derivatives $f^{\prime \prime}(0)$, $-\theta^{\prime}(0)$, and $-\varphi^{\prime}(0)$ are computed and provided in Table 3.

Table 3 shows that the values of skin friction will grow if any values of $\delta, E_{c}, \varepsilon, K_{p}$ and $R_{d}$ are increased, or any values of $N_{r}, Q^{-}$and $\gamma^{-}$are decreased. The local Nusselt number can be increased by increasing $N_{r}, \gamma$ and $Q^{-}$or decreasing $E_{c}, \varepsilon$ and $K_{p}$. Also, the local Sherwood number can be 


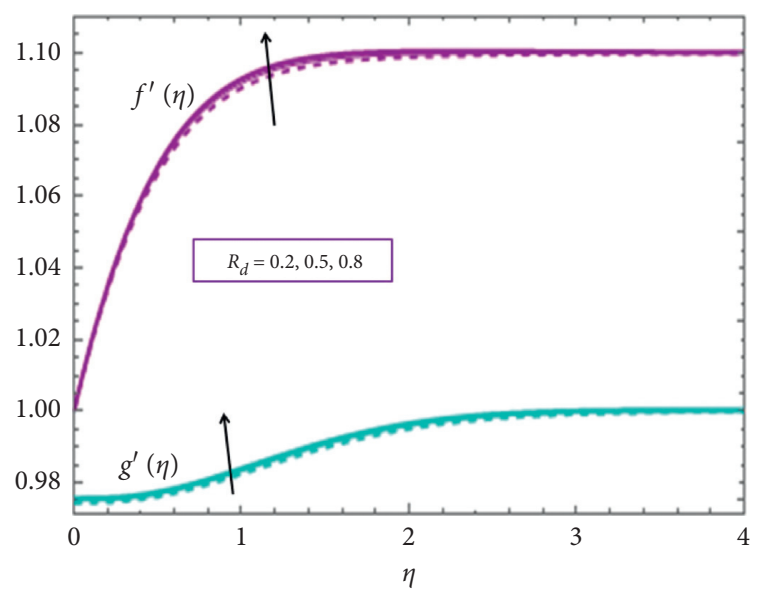

(a)

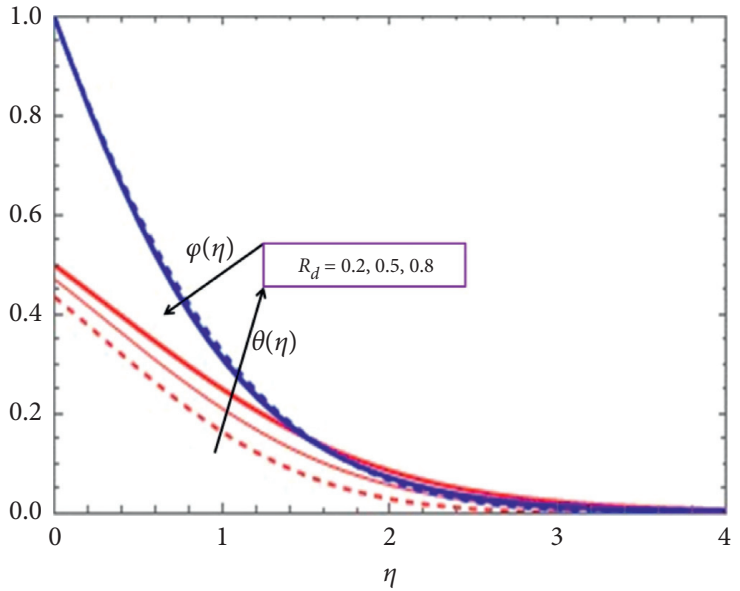

(b)

Figure 11: (a) Effects of thermal radiation parameter $R_{d}$ on velocity and induced magnetic field profiles. (b) Effects of thermal radiation parameter $R_{d}$ on temperature and concentration profiles.

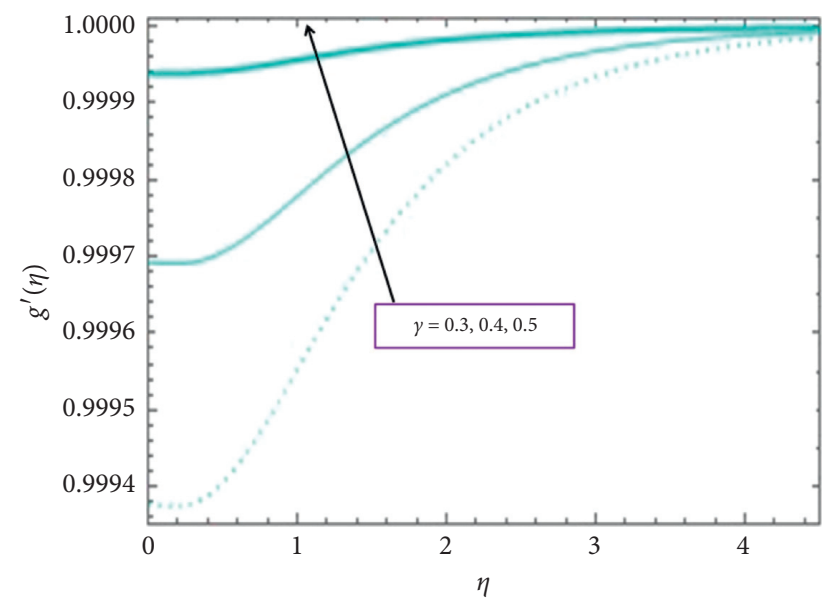

(a)

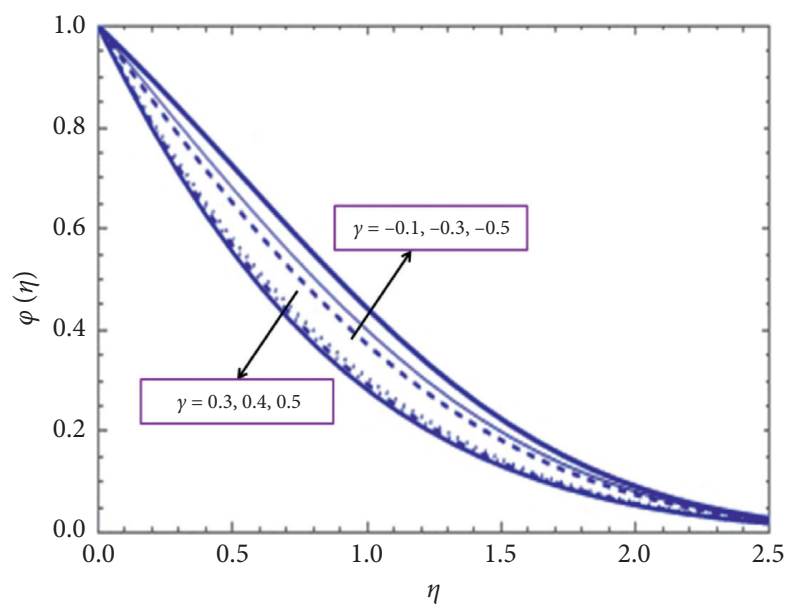

(b)

FIGURE 12: (a) Effects of chemical reaction on induced magnetic field profile. (b) Effects of chemical reaction on concentration profile.

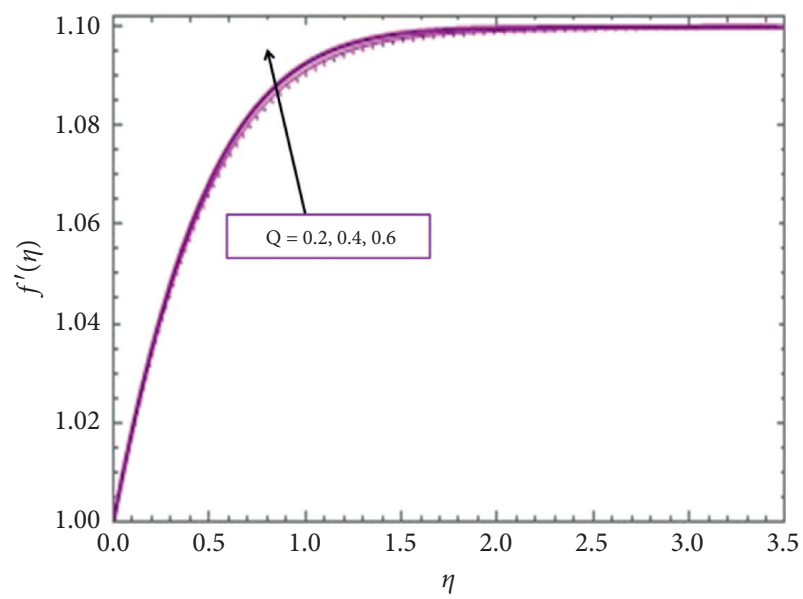

(a)

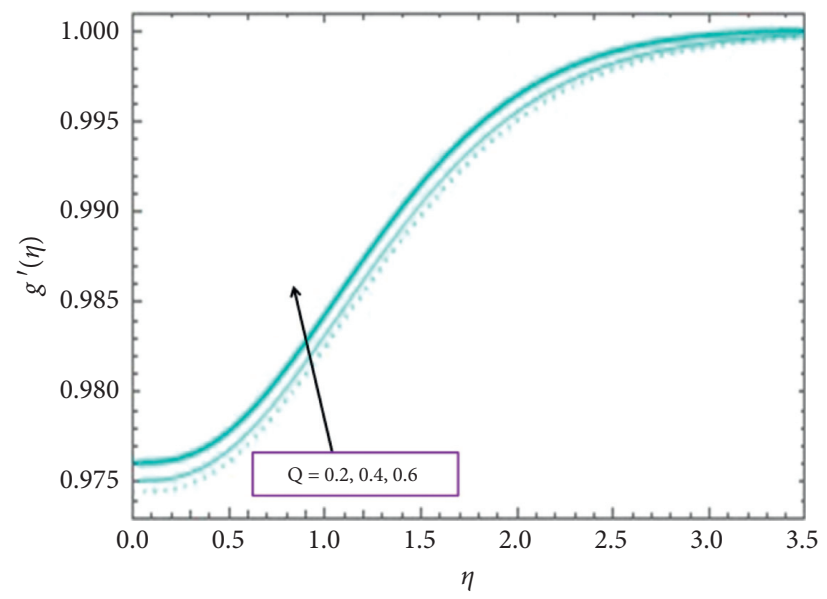

(b)

FIGURE 13: Continued. 


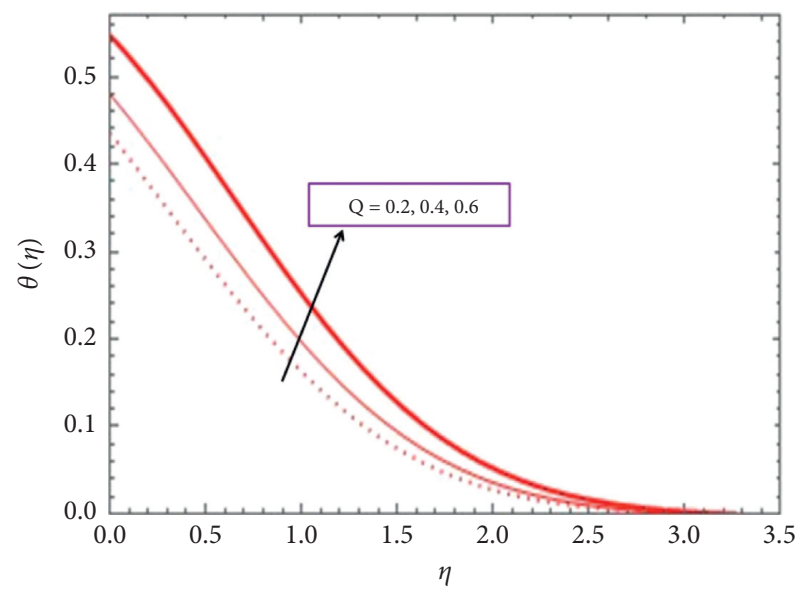

(c)

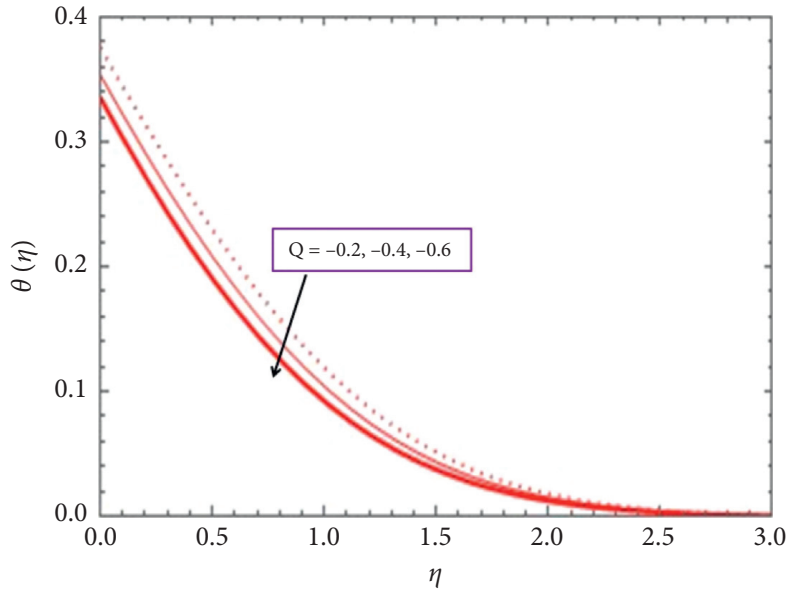

(d)

Figure 13: (a) Effects of heat generation on velocity profile. (b) Effects of heat generation on induced magnetic field profile. (c) Effects of heat generation parameter on temperature profile. (d) Effects of heat absorption parameter on temperature profile.

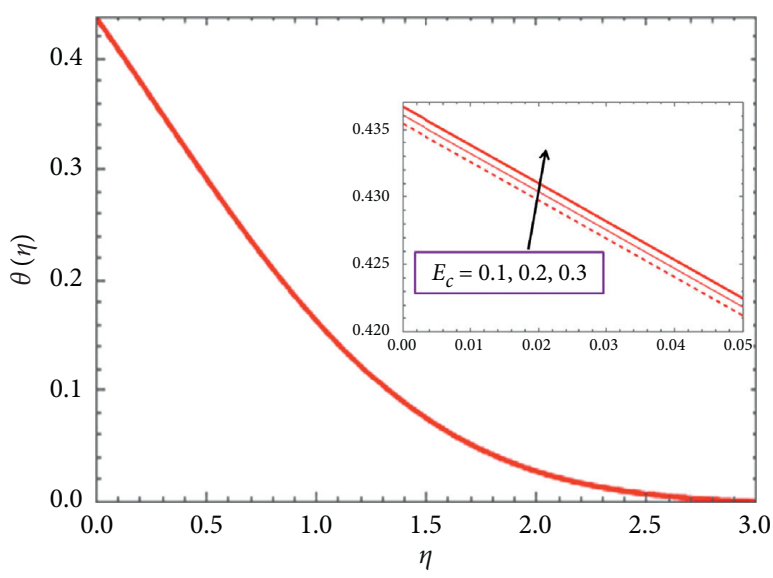

(a)

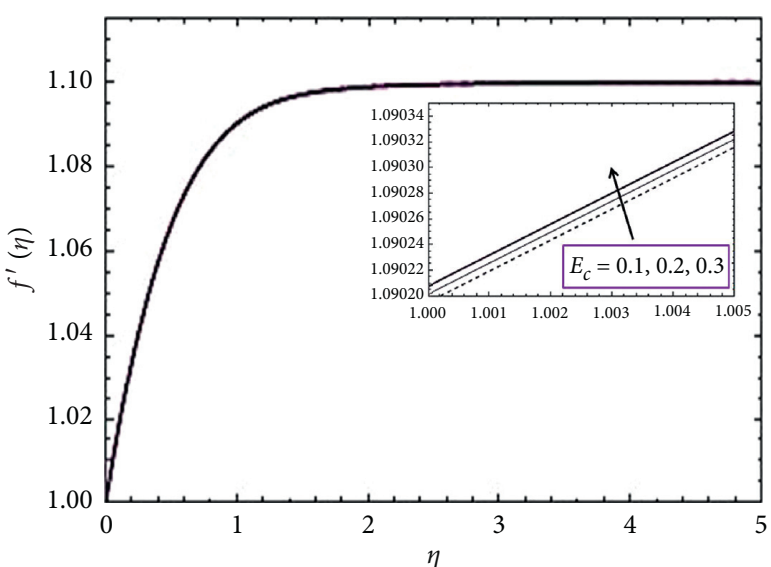

(b)

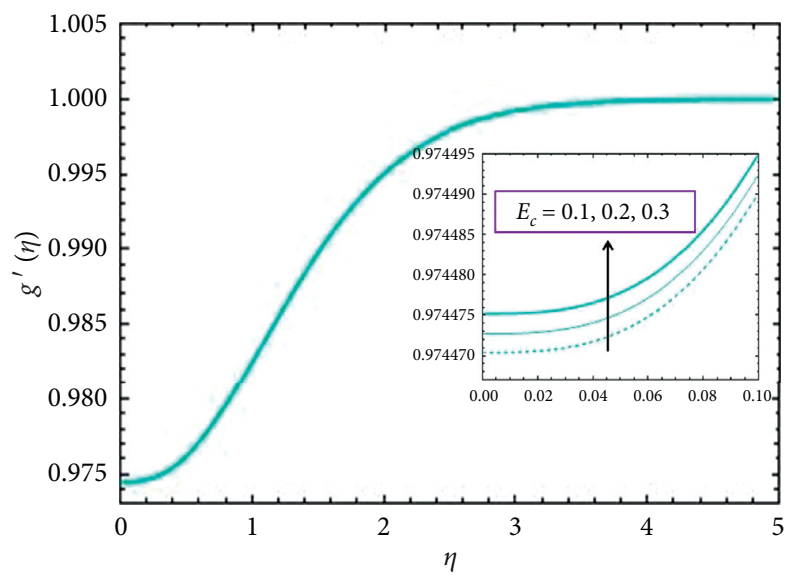

(c)

FIGURE 14: (a) Impacts of Eckert number on temperature profile. (b) Impacts of Eckert number on velocity profile. (c) Impacts of Eckert number on induced magnetic field profile. 


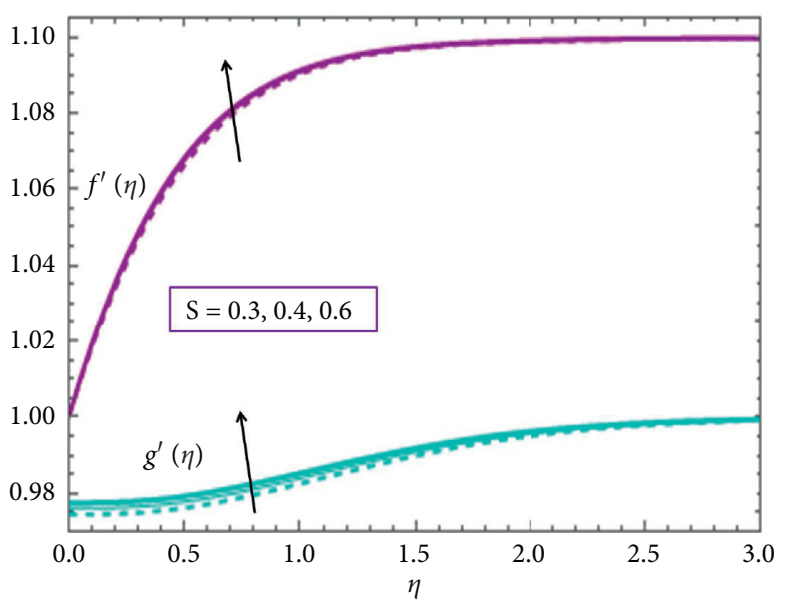

(a)

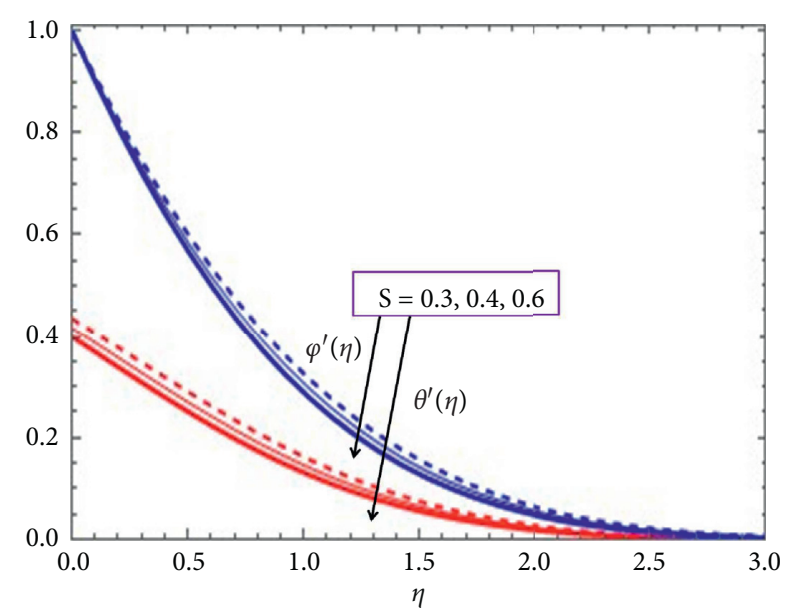

(b)

FIgURE 15: (a) Effects of $S$ on velocity and induced magnetic field. (b) Effects of $S$ on temperature and concentration profiles.

TABle 3: Coefficients of skin friction, Nusselt number, and Sherwood number.

\begin{tabular}{|c|c|c|c|c|c|c|c|c|c|c|c|}
\hline$\delta$ & $N_{r}$ & $E_{c}$ & $\varepsilon$ & $K_{p}$ & $R_{d}$ & $\mathrm{~S}$ & $\gamma$ & $\mathrm{Q}$ & $f^{\prime \prime}(0)$ & $-\theta^{\prime}(0)$ & $-\varphi^{\prime}(0)$ \\
\hline 1.0 & & & & & & & & & 0.01547 & 0.28788 & 1.02624 \\
\hline 1.5 & & & & & & & & & 1.10046 & 0.29696 & 1.11604 \\
\hline \multirow[t]{23}{*}{2.0} & 0.1 & & & & & & & & 2.40058 & 0.28207 & 1.23935 \\
\hline & 1.5 & & & & & & & & 2.34157 & 0.28291 & 1.23463 \\
\hline & 3.0 & 0.1 & & & & & & & 2.29209 & 0.28348 & 1.23162 \\
\hline & & 0.2 & & & & & & & 2.29454 & 0.25120 & 1.29054 \\
\hline & & 0.3 & 1.5 & & & & & & 2.29700 & 0.21874 & 1.34976 \\
\hline & & & 3.0 & & & & & & 2.29998 & 0.21868 & 1.34996 \\
\hline & & & 4.5 & 0.2 & & & & & 2.30274 & 0.21853 & 1.35028 \\
\hline & & & & 0.4 & & & & & 2.34923 & 0.21693 & 1.35473 \\
\hline & & & & 0.6 & 0.2 & & & & 2.39478 & 0.21536 & 1.35907 \\
\hline & & & & & 0.5 & & & & 2.39643 & 0.21755 & 1.33832 \\
\hline & & & & & 0.8 & 0.3 & & & 2.39779 & 0.21723 & 1.32524 \\
\hline & & & & & & 0.4 & & & 2.39779 & 0.21723 & 1.32524 \\
\hline & & & & & & 0.5 & 0.3 & & 2.47838 & 0.22208 & 1.38516 \\
\hline & & & & & & & 0.4 & & 2.55919 & 0.22701 & 1.48693 \\
\hline & & & & & & & 0.5 & 0.2 & 1.05818 & 0.29624 & 1.01902 \\
\hline & & & & & & & & 0.4 & 1.03638 & 0.28853 & 0.96644 \\
\hline & & & & & & & & 0.6 & 1.03638 & 0.28853 & 0.96644 \\
\hline & & & & & & & & -0.2 & 2.55751 & 0.24985 & 1.49336 \\
\hline & & & & & & & & -0.4 & 2.55644 & 0.25935 & 1.47937 \\
\hline & & & & & & & & -0.6 & 2.55523 & 0.26784 & 1.46680 \\
\hline & & & & & & & -0.1 & & 2.54862 & 0.26839 & 1.21123 \\
\hline & & & & & & & -0.3 & & 2.54607 & 0.26865 & 1.11602 \\
\hline & & & & & & & -0.5 & & 2.54326 & 0.26895 & 1.01462 \\
\hline
\end{tabular}

maximized by increasing $B, E_{c}, \varepsilon$ and $K_{p}$ or reducing $N_{r}, R_{d}, Q^{-}$and $\gamma^{-}$.

\section{Concluding Remarks}

In this study, the heat and mass transfer phenomena in MHD stagnation point flow of Maxwell nanofluid towards a convectively heated, vertical, and stretching sheet embedded in a porous medium have been analyzed in the presence of induced magnetic field and various thermophysical parameters. The homotopy analysis method (HAM) has been applied to obtain series solutions for the steady twodimensional laminar flow near the stagnation point. Convergence of the method has been verified. Further, the results obtained in this study are also found in excellent agreement with previous study works under some restricted assumptions. The influences of pertinent parameters on velocity, induced magnetic field, temperature, and concentration profiles are examined as summarized below:

(i) The flow velocity can be accelerated by increasing rates of stretching, external magnetic field, Maxwell parameter, convective parameter, reciprocal of 
Prandtl number, radiation, porosity, dissipation, heat source, suction, or constructive chemical reaction. It can also be increased by reducing the effects of buoyancy ratio or destructive chemical reaction.

(ii) The induced magnetic field profile can be enhanced by increasing the values of external magnetic field, Maxwell parameter, reciprocal of Prandtl number, radiation, porosity, dissipation parameter, heat source, suction, or constructive chemical reaction. It can also be increased by reducing the effects of buoyancy ratio or stretching parameter.

(iii) The temperature profile can be strengthened near the stagnation point by increasing the values of Maxwell parameter, thermal radiation, buoyancy force, heat source, dissipation parameter, or destructive chemical reaction. The temperature can also be maximized by minimizing the effects of external magnetic field, convective parameter, reciprocal of Prandtl number, porosity of the medium, permeability of the sheet, heat sink, constructive chemical reaction, or stretching parameter.

(iv) The concentration profile of nanoparticles in the region of stagnation point can rise by increasing the buoyancy ratio, heat sink, or constructive chemical reaction. This profile can also be enhanced by decreasing the stretching parameter, radiation, convective parameter, suction, or destructive chemical reaction.

(v) The rate of momentum transfer can be facilitated mainly by increasing the rate of stretching, dissipation parameter, reciprocal of Prandtl number, porosity of the medium, or radiation parameter; it can also be sped up by reducing the effects of buoyancy ratio, heat sink or destructive chemical reaction.

(vi) Heat transfer rate can be enhanced by increasing the buoyancy ratio, chemical reaction, or heat sink. This transfer can also be facilitated by decreasing the dissipation parameter, reciprocal of Prandtl number, or porosity of the medium.

(vii) Mass transfer rate can be sped up by increasing dissipation parameter, reciprocal of Prandtl number, or porosity of the medium. It can also be facilitated by decreasing the effect of buoyancy ratio, radiation, heat sink, or destructive chemical reaction.

\section{Nomenclature}

$B: \quad$ Magnetic field strength (Nm/A)

$B_{i}$ : $\quad$ Biot number

$B_{0}$ : Constant magnetic field ( $\left.\mathrm{Nm} / \mathrm{A}\right)$

$C$ : Concentration in boundary layer

$C_{f}: \quad$ Skin friction coefficient

$C_{p}: \quad$ Heat capacity $(\mathrm{J} /(\mathrm{KgK}))$
$C_{w}: \quad$ Wall nanoparticle concentration

$C_{\infty}$ : Concentration in ambient flow

$D_{B}$ : Brownian diffusion coefficient $\left(\mathrm{m}^{2} / \mathrm{s}\right)$

$D_{T}$ : Thermophoretic diffusion coefficient

$\mathrm{E}:{ }_{c}:$ Eckert number

$f:$ Dimensionless stream function

$g: \quad$ Gravitational acceleration

$H$ : $\quad$ Induced magnetic field

$h_{f}$ : Convective heat transfer $\mathrm{W} /\left(\mathrm{m}^{2} \cdot \mathrm{K}\right)$

$J_{w}$ : Mass flux at the surface

$K_{0}$ : $\quad$ Permeability of porous medium

$K_{P}$ : Porosity parameter

$K_{r}$ : $\quad$ Rate of chemical reaction

$k^{*}$ : Thermal absorption coefficient

$M: \quad$ Magnetic field strength

$N_{b}$ : Brownian motion parameter

$N_{t}$ : Thermophoresis parameter

$N_{r}$ : Buoyancy ratio

$\mathrm{Nu}_{x}$ : Nusselt number

$P_{r}: \quad$ Prandtl number

Q: $\quad$ Heat generation/absorption parameter

$Q_{0}: \quad$ Coefficient of heat source

$q_{m}$ : Mass flux of the nanofluid $\left(\mathrm{Kgm}^{2} \mathrm{~s}\right)$

$q_{w}: \quad$ Surface heat flux $\left(\mathrm{W} / \mathrm{m}^{2}\right)$

$r$ : $\quad$ Gravity dependent parameter

Rd: Radiation parameter

$\operatorname{Re}_{x}$ : Local Reynolds number

$S: \quad$ Injection and suction parameter

$\mathrm{Sh}_{x}$ : Sherwood number

$S_{c}: \quad$ Schmidt number

$T: \quad$ Temperature (K)

$T_{f}$ : Temperature of heated fluid (K)

$T_{\infty}$ : Ambient fluid temperature (K)

$U_{e}: \quad$ Ambient velocity $(\mathrm{m} / \mathrm{s})$

$U_{w}: \quad$ Surface velocity $(\mathrm{m} / \mathrm{s})$

$V_{w}$ : Velocity of mass through the wall $(\mathrm{m} / \mathrm{s})$

$\alpha$ : Thermal diffusivity of the nanofluid

$\beta_{0}$ : Thermal expansion

$\gamma: \quad$ Chemical reaction parameter

$\delta: \quad$ Velocity ratio

$\varepsilon$ : Magnetic diffusivity number

$\eta$ : Similarity variable

$\theta$ : Dimensionless temperature

$\kappa: \quad$ Thermal conductivity $(\mathrm{W} /(\mathrm{mK}))$

$\lambda$ : Deborah number

$\lambda_{0}$ : Coefficient of viscoelasticity

$\mu: \quad$ Dynamic viscosity $(\mathrm{Kg} /(\mathrm{m} \cdot \mathrm{s}))$

$\mu_{m}: \quad$ Magnetic permeability

$\rho_{f}: \quad$ Density of fluid $\left(\mathrm{Kg} /\left(\mathrm{m}^{3}\right)\right)$

$\rho_{p}: \quad$ Density of nanoparticles $\left(\mathrm{Kg} /\left(\mathrm{m}^{3}\right)\right)$

$(\rho c)_{f}$ : Heat capacity of the fluid

$(\rho c)_{p}$ : Heat capacity of the nanoparticles

$\sigma: \quad$ Electric conductivity

$\sigma^{*}: \quad$ Stefan-Boltzmann constant

$\tau$ : $\quad$ Ratio of heat capacities

$\tau_{w}: \quad$ Wall shear stress $(\mathrm{Pa})$

$v$ : Kinematic viscosity $\left(\mathrm{m}^{2} / \mathrm{s}\right)$

$\varphi: \quad$ Dimensionless concentration function 
$\phi$ : Homotopy approximation

$\psi: \quad$ Stokes stream function

$\hbar$ : Convergence-control parameter.

\section{Data Availability}

No supporting data were considered in this study.

\section{Conflicts of Interest}

The authors declare that they have no conflicts of interest.

\section{References}

[1] U. S. Choi and J. A. Eastman, Enhancing Thermal Conductivity of Fluids with Nanoparticles, Argonne National Laboratory, Lemont, IL, USA, 1995.

[2] W. Ibrahim and A. Tulu, "Magnetohydrodynamic (MHD) boundary layer flow past a wedge with heat transfer and viscous effects of nanofluid embedded in porous media," Mathematical Problems in Engineering, vol. 2019, Article ID 4507852, 12 pages, 2019.

[3] L. Zhang, M. B. Arain, M. M. Bhatti, A. Zeeshan, and H. HalSulami, "Effects of magnetic Reynolds number on swimming of gyrotactic microorganisms between rotating circular plates filled with nanofluids," Applied Mathematics and Mechanics, vol. 41, no. 4, pp. 637-654, 2020.

[4] M. B. Arain, M. M. Bhatti, A. Zeeshan, S. Tareq, and H. Aatef, "Analysis of arrhenius kinetics on multiphase flow between a pair of rotating circular plates," Mathematical Problems in Engineering, vol. 2020, Article ID 2749105, 17 pages, 2020.

[5] F. A. Alwawi, H. T. Alkasasbeh, A. Rashad, and R. Idris, "Heat transfer analysis of ethylene glycol-based Casson nanofluid around a horizontal circular cylinder with MHD effect," Proceedings of the Institution of Mechanical Engineers, Part C: Journal of Mechanical Engineering Science, vol. 234, no. 13, pp. 2569-2580, 2020.

[6] V. Nagendramma, K. Kumar, D. Prasad, A. Leelaratnam, and K. Varma, "Multiple slips and thermophoresis effects of Maxwell nanofluid over a permeable stretching surface in the presence of radiation and dissipation," Journal of Nanofluids, vol. 5, pp. 1-9, 2016.

[7] G. K. Ramesh, B. J. Gireesha, T. Hayat, and A. Alsaedi, "Stagnation point flow of Maxwell fluid towards a permeable surface in the presence of nanoparticles," Alexandria Engineering Journal, vol. 55, no. 2, pp. 857-865, 2016.

[8] Y. Bai, X. Liu, Y. Zhang, and M. Zhang, "Stagnation-point heat and mass transfer of MHD Maxwell nanofluids over a stretching surface in the presence of thermophoresis," Journal of Molecular Liquids, vol. 224, pp. 1172-1180, 2016.

[9] A. Haritha, Y. Devasena, and B. Vishali, "MHD heat and mass transfer of the unsteady flow of a Maxwell fluid over a stretching surface with Navier slip and convective boundary conditions," Global Journal of Pure and Applied Mathematics, vol. 13, no. 6, pp. 2169-2179, 2017.

[10] A. Mushtaq, M. Mustafa, T. Hayat, and A. Ahmed, "Buoyancy effects in stagnation-point flow of Maxwell fluid utilizing non-Fourier heat flux approach," PLoS One, vol. 13, no. 5, Article ID e0192685, 2018.

[11] M. Khan, Y. Malik, T. Salahuddin, and F. Khan, "Generalized diffusion effects on Maxwell nanofluid stagnation point flow over a stretchable sheet with slip conditions and chemical reaction," Journal of the Brazilian Society of Mechanical Sciences and Engineering, vol. 41, pp. 138-147, 2019.

[12] A. Aziz and M. Shams, "Entropy generation in MHD Maxwell nanofluid flow with variable thermal conductivity, thermal radiation, slip conditions, and heat source," AIP Advances, vol. 10, pp. 1-12, 2020.

[13] W. Ibrahim, "The effect of induced magnetic field and convective boundary condition on MHD stagnation point flow and heat transfer of upper-convected Maxwell fluid in the presence of nanoparticle past a stretching sheet," Propulsion and Power Research, vol. 5, no. 2, pp. 164-175, 2016.

[14] T. Hayat and S. Nadeem, "Induced magnetic field stagnation point flow of nanofluid past convectively heated stretching sheet with Buoyancy effects," Chinese Physics B, vol. 25, no. 11, Article ID 114701, 2016.

[15] J. Liao, Beyond Perturbation: Introduction to Homotopy Analysis Method, Chapman and Hall/CRC, Boca Raton, FL, USA, 2003.

[16] S. Liao, "Homotopy analysis method in nonlinear differential equations," Advances in the Homotopy Analysis method, vol. 361, 2012.

[17] Y. Zhao and S. J. Liao, Advances in the Homotopy Anal Method, p. 361, World Scientific Publishing Co. Pte. Ltd., Singapore, 2013. 